\title{
Macrophage CCL22 expression promotes lymphangiogenesis in patients with tongue squamous cell carcinoma via IL-4/STAT6 in the tumor microenvironment
}

\author{
SATOSHI KIMURA ${ }^{1,2}$, HIROTSUGU NOGUCHI ${ }^{2}$, UKI NANBU ${ }^{2}$ and TOSHIYUKI NAKAYAMA ${ }^{2}$ \\ ${ }^{1}$ Department of Clinical Pathology, Kitakyushu City Yahata Hospital, Kitakyushu, Fukuoka 805-8534; \\ ${ }^{2}$ Department of Pathology, School of Medicine, University of Occupational and Environmental Health, \\ Kitakyushu, Fukuoka 807-8555, Japan
}

Received October 21, 2020; Accepted February 15, 2021

DOI: $10.3892 / \mathrm{ol} .2021 .12644$

\begin{abstract}
The C-C motif chemokine ligand 22 (CCL22) chemokine is produced by M2-like tumor-associated macrophages (TAMs) in the tumor microenvironment. Chemokine C-C motif receptor 4 (CCR4), the CCL22 receptor, on $\mathrm{T}$ helper2 (Th2) cells leads to a Th2 cytokine-dominant environment. In our previous study, lymph node metastasis was the main predictor of tongue squamous cell carcinoma (SCC) via CCL22. Therefore, the present study aimed to investigate the effects of CCL22 and a Th2 cytokine-predominant tumor microenvironment on vascular endothelial growth factor (VEGF)-C expression and lymphangiogenesis. The post-operative courses of 110 patients with early-stage tongue SCC with a histopathological diagnosis based on the 8th TNM classification were followed up (mean/median follow-up time, 47.1/42.0 months) from surgery until death or the last follow-up visit, and subsequent lymph node relapse was assessed. Lymphangiogenesis and the immunohistochemical expression of several markers (CCL22, CCR4 and VEGF-C) were evaluated. The Kaplan-Meier method was used to plot lymph node relapse-free survival and overall survival curves, which were compared using the log-rank test. In vitro, the association between CCL22 and VEGF-C by interleukin (IL)-4/signal
\end{abstract}

Correspondence to: Dr Satoshi Kimura, Department of Clinical Pathology, Kitakyushu City Yahata Hospital, 2-6-2 Ogura, Yahatahigashi, Kitakyushu, Fukuoka 805-8534, Japan

E-mail: s-kimura@med.uoeh-u.ac.jp

Abbreviations: TAM, tumor-associated macrophage; CCR4, chemokine C-C motif receptor 4; SCC, squamous cell carcinoma; VEGF, vascular endothelial growth factor; IL-4, interleukin-4; STAT6, signal transducer and activator of transcription 6; CTL, cytotoxic T cells; LNFS, lymph node relapse-free survival; OS, overall survival; CCL22, C-C motif chemokine 22; WPOI, worst pattern of invasion; DOI, depth of invasion

Key words: tongue SCC, tumor microenvironment, chemokine, CCL22, macrophage, STAT6, WPOI, DOI transducer and activator of transcription 6 (STAT6) stimulation was examined. Lymphangiogenesis was significantly associated with lymph node relapse $(\mathrm{P}<0.001)$ and a CCL22 ${ }^{+}$ macrophage ratio $(\mathrm{P}<0.001)$. CCL22 $2^{+}$TAMs were positive for VEGF-C and surrounded by $\mathrm{CCR} 4^{+}$cells. Additionally, VEGF-C expression was increased in IL-4/STAT6-stimulated macrophages. In addition, the STAT6 signaling pathway was activated in the SCC cells in the deeply invaded part of the tumor along with the aggregated macrophages. In conclusion, TAM CCL22 expression led to lymph node relapse via VEGF-C expression within the tumor microenvironment and the IL-4/STAT6 signaling pathway in early stage tongue SCC. Additionally, the worst pattern of invasion and depth of invasion were revealed to be useful parameters for lymph node relapse in patients with tongue SCC. The present study suggested that CCL22 contributed to the role of M2-like differentiated TAMs in prognosis and lymph node relapse via IL-4/STAT6 and VEGF. The IL-4/STAT6 signaling pathway may be a new molecular target for tongue SCC.

\section{Introduction}

Squamous cell carcinoma (SCC) of the tongue is a common oral malignancy affecting patients worldwide (1-3). Lymph node metastasis of the neck is an important prognostic factor for this disease $(4,5)$. In our previous study of early SCC of the tongue, post-operative lymph node relapse was also identified as an important prognostic factor (6).

In recent years, novel immunotherapies have received much attention. However, the mechanisms involved in their anticancer effects are still not fully understood. In addition, it has been suggested that chemokines may also influence mechanisms within the tumor microenvironment (7), which is composed of many immune and inflammatory cells, including macrophages. M2-like macrophages, in particular, are major constituent cells and an important source of chemokines (8).

A pivotal chemokine, CCL22 is mainly expressed by macrophages and dendritic cells (9), while its receptor, chemokine $\mathrm{C}-\mathrm{C}$ motif receptor 4 (CCR4), is mainly expressed by $\mathrm{T}$ cells (10). With regard to the tumor microenvironment, CCL22 is expressed mainly by M2-like tumor-associated 
macrophages (TAMs) (6). CCR4 is also known as a marker of regulatory $\mathrm{T}$ cells (Tregs) and Th2 cells (11,12). CCL22 attracts Th2 cells via CCR4 increasing Th2 cytokines [e.g., interleukin (IL)-4], which leads to the increased expression of CCL22 in M2-like macrophages $(12,13)$. Cytotoxic $\mathrm{T}$ cells (CTLs) are anti-tumor immune cells that are also found in the tumor microenvironment (14). The expression of CCL22 was suggested to be involved in the suppression of CTLs in tongue SCCs via the expression of Tregs (6).

Several studies have revealed that vascular endothelial growth factor (VEGF)-C leads to lymphangiogenesis and the induction of lymph node metastasis $(15,16)$. CD163, one of the representative markers of M2-like macrophages (17), is involved in the expression of VEGF-C in oral SCC (18). Since CD163 is expressed in the same macrophages as CCL22 $(6,19)$, it follows that whether CCL22 expression is involved in VEGF-C expression and lymphangiogenesis should be investigated. In addition, several head and neck SCCs express CCR4, which, together with CCL22, allow carcinoma cells to migrate to lymph nodes (20).

In the diagnosis of tongue SCC, it should be noted that characterizing an invasive pattern is useful for a prognosis and to predict lymph node metastasis $(6,21)$. The worst pattern of invasion (WPOI) is one such example. The WPOI, originally described in 2005, sets out various grades: type 1 refers to a pushing border; type 2 is a finger-like growth, type 3 refers to large separate islands with more than 15 cells per island; type 4 represents small tumor islands with 15 cells or fewer per island; and type 5 represents a satellite of the tumor, $\geq 1 \mathrm{~mm}$ from the main tumor or the next closest satellite (22). The WPOI mode of invasion was found to significantly correlate and affect the prognosis of OSCC (23).

More recently, the depth of invasion (DOI) has also attracted attention as a prognostic factor (24) and is associated with lymph node metastasis (25). According to the new 8th TNM classification, DOI determines pathological or clinical T staging (26).

In the current study, the relationship between the expression of CCL22 in the tumor microenvironment and lymph node relapse in patients with tongue SCC, with reference to WPOI and DOI, was investigated.

\section{Materials and methods}

Patient cohort and study design. We examined tumor sections from 110 patients with tongue SCC who underwent primary surgery at a hospital at the University of Occupational and Environmental Health, Kitakyushu, Japan, between January 1997 and August 2017. Patients in this study had the following background: Tumor size $\leq 40 \mathrm{~mm}$; depth of invasion $\leq 10 \mathrm{~mm}$; no metastasis in any node; surgical margins $>5 \mathrm{~mm}$; and had not undertaken neoadjuvant therapy prior to surgery. Lymph node metastasis was monitored using imaging analyses (ultrasonography, computed tomography, or magnetic resonance imaging) in 2- to 6-month intervals post-surgery and verified by pathological diagnosis. Lymph node relapse-free survival (LNFS) and overall survival (OS), as the endpoints of analysis (September 2019), were defined as the time from surgery until lymph node relapse or death had occurred, respectively, or until the last follow-up visit.
Patient samples (females, 32; males, 78) aged 32-89 years [mean \pm standard deviation (SD), 64.6 \pm 13.3 years] were obtained at diagnosis.

Our study was approved by the Research Ethics Committee of the University of Occupational and Environmental Health (permission nos. H29-212 and H24-6) and was performed in accordance with the guidelines of the Declaration of Helsinki.

Histopathological staining and classification of tongue SCC, WPOI, and histological grade. After fixation of resected tumor tissues in $10 \%$ formalin and embedding in paraffin, sections (thickness, $3 \mu \mathrm{m}$ ) underwent hematoxylin and eosin (H\&E) staining.

Histological classification was based on the American Joint Committee on Cancer Staging Manual, 8th edition (27). The WPOI classification system was used to assess the pattern of tumor invasion in patients. Tumor differentiation was divided into three groups: well-differentiated (I), moderately differentiated (II), and poorly differentiated (III; Table I).

Immunohistochemical studies. An automated immunostainer, Histostainer 36A (Nichirei Biosciences Inc.), was used to process sections that had been fixed in $10 \%$ formalin and immersed in paraffin in accordance with the manufacturer's protocol. Antibodies used were: mouse monoclonal anti-human CD68 (clone Kp-1, 1:100) and CD8 (clone C8/144B, 1:100), both from Dako Japan; and rabbit polyclonal antibody to CCL22 (1:100) and goat polyclonal antibody to CCR4 (1:300), both from Abcam. Mouse monoclonal anti-human VEGF-C (clone MM0006-2E65, 1:50) was obtained from Novus Biologicals. Mouse monoclonal anti-human antibody to D2-40 (clone D2-40, 1:1; Nichirei) was used to examine lymphatic vessels.

Five areas were randomly selected and CCL22-, CCR4$\mathrm{CD}^{-}$, and VEGF-C-positive cells were counted using a microscope (magnification, $\mathrm{x} 400$ ); data was expressed as per $\mathrm{mm}^{2}$ surface area. Two independent pathologists, blinded to patients' backgrounds or their prognosis, evaluated all immunochemical and histological slides.

Cell proliferation assays were also performed, as described in our previous study, by using anti-Ki67 (mouse monoclonal, clone MIB-1; 1:100; Dako) (6).

Agreement among observers was excellent (agreement $>95 \%$ ) for all antibodies and according to an interclass correlation coefficient. In the case of a rare disagreement, a third, departmental board-certified pathologist calculated a consensus score.

Immunofluorescence assays. Thin sections ( $4 \mu \mathrm{m})$ were used for immunofluorescence assays and anti-CCL22, CCR4 and VEGF-C antibodies were used in experiments as described above. In addition, we used rabbit polyclonal anti-human VEGF-C (1:100; Santa Cruz Biotechnology, Inc.), and rabbit polyclonal anti-human signal transducer and activator of a transcription 6 (STAT6; phospho Y641) (1:100; Abcam). Secondary antibodies used were: rhodamine-conjugated donkey anti-rabbit IgG (1:200; Merck) and goat anti-mouse IgG (H+L; 1:200; Merck); and FITC-conjugated goat anti-rabbit IgG (H+L; 1:200; Merck) and donkey anti-goat IgG (1:200; Merck). A nuclear stain, 4',6-diamidino-2-phenylindole 
(DAPI; GeneTex), was used on sections, which were then mounted. An ECLIPSE E600 inverted fluorescence microscope (Nikon) was used to examine slides, and images captured and analyzed with LuminaVision software (v. 2.2.2; Mitani Corporation).

Quantification of lymphangiogenesis. Using anti-D2-40, the density of lymphatic vessels (LVD) was measured as previously described (28). LVD was evaluated at the periphery, within $2 \mathrm{~mm}$ of the tumor, and next to the invasive front. Five areas that showed many lymphatic vessels were chosen using light microscopy at a x40 magnification. All stained vessels in each area at a x200 magnification were counted and the data was expressed as per $\mathrm{mm}^{2}$. The mean number of lymphatic vessels was calculated and expressed as LVD in a blinded manner (described above).

Cell culture. A human macrophage cell line (human CD14 ${ }^{+}$ monocytes from peripheral blood, single donor; C-12909, https://www.promocell.com/product/human-cd14-mono-

cytes-hmocd14-pb/) from PromoCell GmbH (Heidelberg, Germany) was cultured at a density of $1 \times 10^{6}$ cells in Monocyte Attachment Medium (PromoCell GmbH). Cells were cultured in M1-Macrophage Generation Medium DXF so that they could differentiate into M1- and M2-like macrophages as in our previous study (19). In addition, these cells were treated with 1,10 or $20 \mathrm{ng} / \mathrm{ml}$ of interleukin-4 (IL-4; R\&D Systems) for 24 h. Human monocytic (THP-1) cells were obtained from the American Type Culture Collection and maintained in RPMI-1640 containing 10\% fetal bovine serum (Gibco; Thermo Fisher Scientific, Inc.), $1 \%$ penicillin-streptomycin (Gibco; Thermo Fisher Scientific, Inc.), and $2 \mathrm{mmol} / \mathrm{l}$ of glutamine (Gibco; Thermo Fisher Scientific, Inc.).

Real-time polymerase chain reaction. For quantitative real-time polymerase chain reactions (qRT-PCR), a TaqMan assay and a 7700 Sequence Detection System (Applied Biosystems; Thermo Fisher Scientific, Inc.) were used as in our previous study (19). A pre-made primer/probe set (Applied Biosystems Japan, Ltd.) containing primers and fluorogenic probes for CCL22 and VEGFC was used. Messenger RNA expression was normalized to that of $18 \mathrm{~s}$ ribosomal RNA in each sample.

Luciferase reporter assay. The CCL22 gene was cloned from an upstream gene region using information on chromosome $16 q 13$, and a luciferase assay was performed as described in our previous study (29). Genomic DNA was extracted from monocytes isolated from $15 \mathrm{ml}$ whole blood donated from four healthy volunteer donors. A human CCL22 promoter region spanning between -966 and +32 bp from the transcription start site was then generated by PCR. A pGEM-T Easy vector was used to subclone the PCR product. Serial 5 -deletion constructs $(-518 /+32,-491 /+32,-281 /+32)$ were generated by PCR and digestion with appropriate restriction enzymes. Luciferase reporter genes that included a mutation in the STAT6 site of the CCL22 promoter were generated by site-directed mutagenesis (Stratagene) using the following primers: 5'-ATGTGGACAGCACGAGAAGCCCCAGAT-3' for STAT6 mutation, where the underlined nucleotides represented the mutated site.
Twenty micrograms of promoterless pGL3-basic vector or CCL22 promoter luciferase constructs were used to transfect THP-1 monocytic cells treated with $20 \mathrm{ng} / \mathrm{ml} \mathrm{12-O-tetradecanoylphorbol-13-acetate}$ as in our previous study (29). A pSV-beta-galactosidase control plasmid (Promega Corporation) was used as an internal control. A luminometer (Bio-Orbit Oy) was used to measure luminescence.

Secretion and regulation of CCL22 in macrophages. CCL22 protein expression via IL-4 (R\&D Systems), with or without AS1517499 (Axon Medchem BV) as a selective STAT6 inhibitor, in human macrophage cell line (C-12909; PromoCell $\mathrm{GmbH}$ ) culture supernatants was measured using an enzyme-linked immunosorbent assay (ELISA) kit (R\&D Systems) as in a previous study (29).

Statistical analysis. Statistical analyses of data between the groups were performed using one-way ANOVA followed by the Tukey-Kramer post-hoc comparison test. Survival curves were plotted according to a Kaplan-Meier method and compared using a log-rank test. Odds ratios (ORs) and corresponding $95 \%$ confidence intervals were calculated from logistic regression models. Pearson's correlation coefficient was used to assess the association between lymphangiogenesis and other factors.

Two-sidedP-values were used. A P-value $\leq 0.05$ was considered statistically significant. EZR software (Saitama Medical Center, Jichi Medical University, Saitama, Japan) was used for all analyses. EZR software is a graphical user interface for $\mathrm{R}$, a modified version of $\mathrm{R}$ commander (version 1.6-3; The R Foundation for Statistical Computing, Vienna, Austria, version 2.13.0) with statistical functions used in biostatistics (30).

The optimal cut-off values for lymph node relapse (maximizing the sum of sensitivity and specificity) were also analyzed by EZR software using receiver operating characteristic (ROC) curve analysis.

\section{Results}

Clinical and pathological characteristics of 110 patients with tongue SCC. Table I shows clinicopathological features of the 110 patients of this study based on a WPOI classification system. The patients were categorized as follows, and the various parameters were compared: WPOI-1, 19 (17.3\%); WPOI-2, 24 (21.8\%); WPOI-3, 24 (21.8\%); WPOI-4, 29 (26.4\%); and WPOI-5, 14 (12.7\%).

There was a high correlation between lymphatic invasion and WPOI classification (WPOI-1 vs. WPOI-2 vs. WPOI-3 vs. WPOI-4 vs. WPOI-5; 0.0 vs. 12.5 vs. 50.0 vs. $62.1 \%$ vs. 1 $00 \%$, respectively). WPOI-1 and -2 patients with lymph node relapse were $2 / 43(4.7 \%)$ of the patient cohort. In comparison, $16 / 67$ patients (23.9\%) with WPOI-3, -4 , and -5 showed lymph node relapse (Table I). The occurrence of lymph node relapse was significant in WPOI-3, -4 , and -5 cases $(\mathrm{P}=0.008$; Fisher's exact test) suggesting WPOI-3, -4 , and -5 lesions were prone to lymph node relapse. In terms of lymph node relapse rates, WPOI was classified into two distinct groups: WPOI-1 and -2 (WPOI-low); and WPOI-3, -4, and -5 (WPOI-high). 

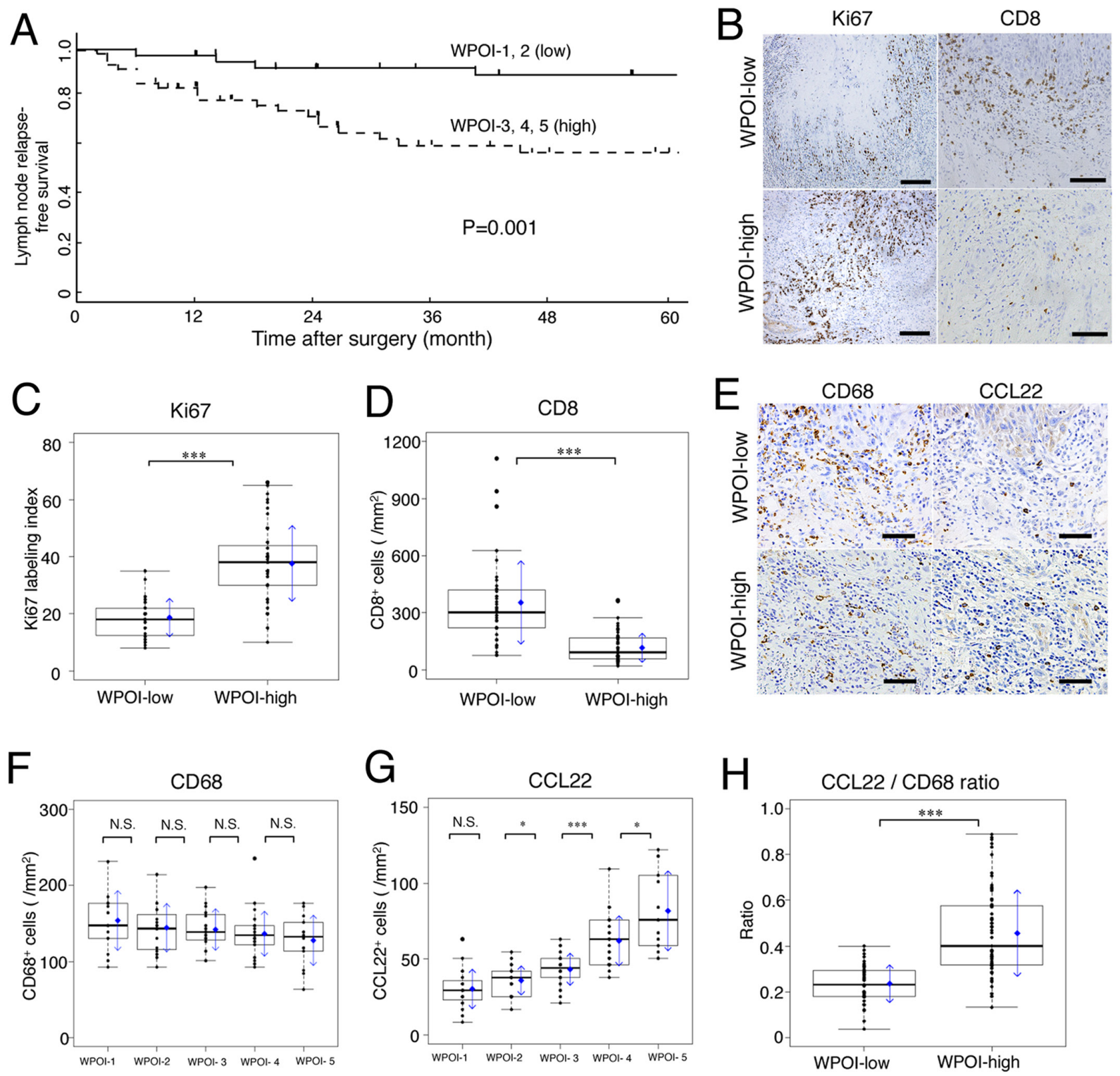

Figure 1. Lymph node relapse-free survival curves for patients with tongue SCC, and association of Ki67, CD8, CD68 and CCL22 with WPOI. WPOI-1 to -5 were classified into two categories based on the frequency of lymph node relapse: WPOI-1 and -2 were considered as WPOI-low, and WPOI-3, -4 and -5 were considered as WPOI-high. (A) Kaplan-Meier lymph node relapse-free survival curves for 110 patients with tongue SCC based on WPOI-low and -high status. (B) Ki67 (scale bar, $200 \mu \mathrm{m}$ ) and CD8 (scale bar, $100 \mu \mathrm{m}$ ) expression in WPOI-low and -high groups by immunohistochemical staining. (C) Ki67 expression was significantly increased and (D) $\mathrm{CD} 8^{+}$cells were significantly decreased in the WPOI-high compared with in the WPOI-low group. (E) CD68 and CCL22 expression in WPOI-low and -high groups by immunohistochemical staining (scale bar, $50 \mu \mathrm{m}$ ). (F) CD68 ${ }^{+}$cells were not significantly different across WPOI classifications, but mean values tended to be inversely proportional to WPOI. (G) CCL22 cells increased proportionally to WPOI classification. (H) Comparison of the ratio between CCL22 ${ }^{+}$cells to CD68 $8^{+}$cells between WPOI-low and -high groups. In box plots, the boxes display the median and interquartile range of the data, and the whiskers display the 10th and 90 th percentiles. Blue diamonds and blue arrows indicate the mean $\pm \mathrm{SD}$. ${ }^{*} \mathrm{P}<0.05$; ${ }^{* * * *} \mathrm{P}<0.001$. N.S., not significant; SCC, squamous cell carcinoma; WPOI, worst pattern of invasion; CCL22, C-C motif chemokine ligand 22.

Impacts of WPOI classification for prognosis. The 5-year LNFS rates in patients in WPOI-low vs. WPOI-high groups were $88.4 \%$ vs. $65.7 \%$, respectively; the difference was statistically significant ( $\mathrm{P}=0.001$; Fig. $1 \mathrm{~A})$. In addition, the 5-year OS rates in patients in WPOI-low vs. WPOI-high groups were 93.6 vs. $76.1 \%$, respectively; the difference was statistically significant $(\mathrm{P}=0.006)$ (data not shown).

Ki67 (a marker of tumor proliferation) was expressed only in the margin of the tumor infiltrating region in WPOI-low and WPOI-high cases; it was randomly expressed in tumor cells of the whole infiltrate (Fig. 1B; Ki67). Scattered CD8-positive cells (cytotoxic T lymphocytes; CTLs) were found in the tumor microenvironment and only a few were found in WPOI-high compared to WPOI-low tissues (Fig. 1B; CD8). WPOI-high tissues had significantly more Ki67-positive tumor cells than WPOI-low tissues ( $\mathrm{P}<0.001$; Fig. 1C). In contrast, CD8-positive cells were significantly decreased in WPOI-high compared to WPOI-low lesions ( $\mathrm{P}<0.001$; Fig. 1D).

In short, the WPOI classification correlated well with the growth potential of the tumor and decreased cellular immunity.

Macrophage expression in tumor microenvironment and relationship with WPOI and lymph node relapse. The 

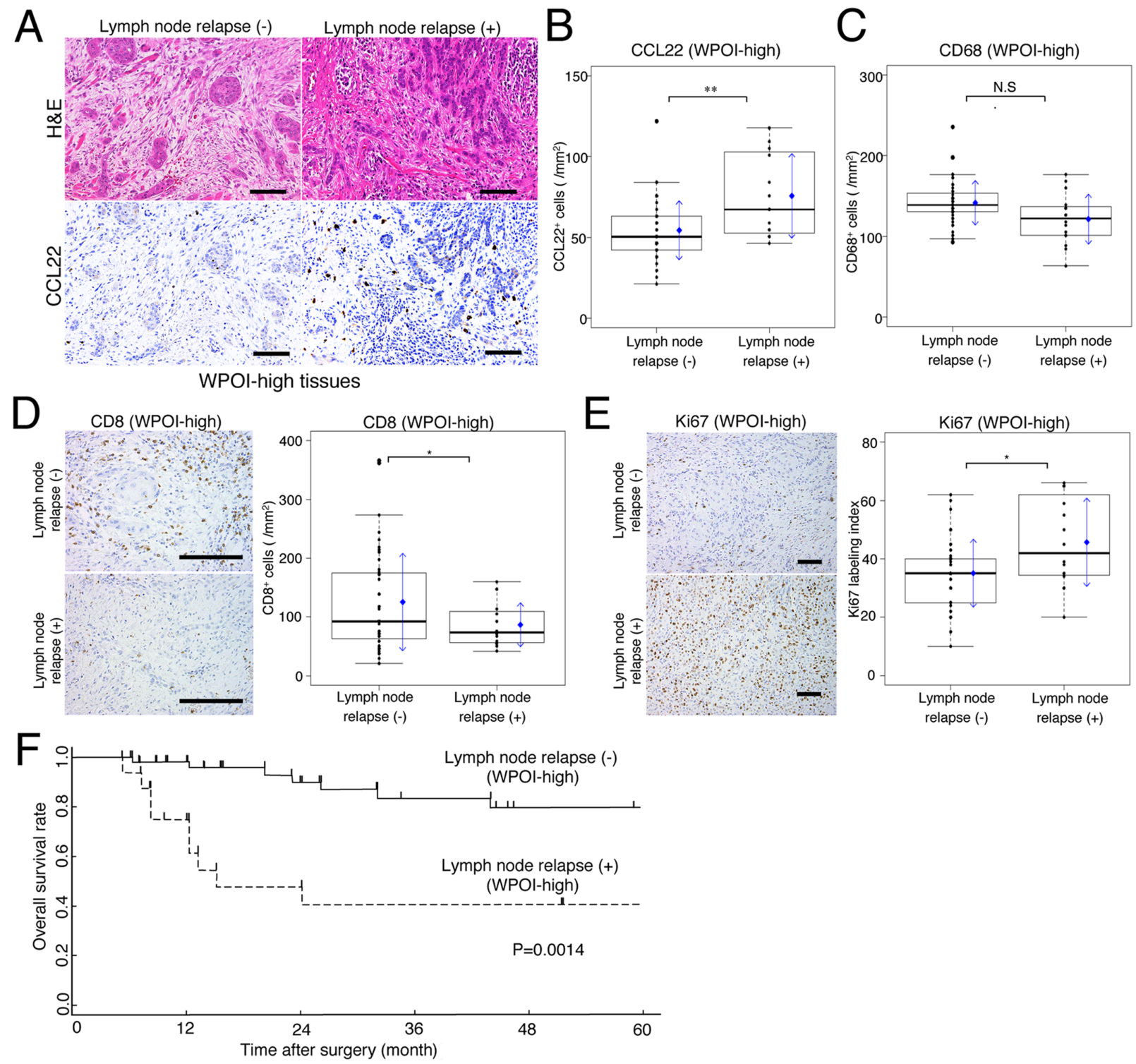

Figure 2. Implications of lymph node relapse for prognosis and CCL22 expression. (A) H\&E and CCL22 immunohistochemical staining of WPOI-high tumor tissues (scale bar, $100 \mu \mathrm{m}$ ). Comparisons of (B) CCL22 $2^{+}$and (C) CD68 $8^{+}$expression in WPOI-high tissues with or without lymph node relapse. Comparisons of (D) CD8 ${ }^{+}$cells and (E) Ki67 expression in WPOI-high tissues with or without lymph node relapse (scale bar, $100 \mu$ m). (F) Kaplan-Meier survival curves in patients with or without lymph node relapse in the WPOI-high group. In box plots, the boxes display the median and interquartile range of the data, and the whiskers display the 10th and 90th percentiles. Blue diamonds and blue arrows indicate the mean $\pm \mathrm{SD} .{ }^{*} \mathrm{P}<0.05 ;{ }^{* * *} \mathrm{P}<0.01$. N.S., not significant; H\&E, hematoxylin and eosin; WPOI, worst pattern of invasion; CCL22, C-C motif chemokine ligand 22.

number of CD68-positive macrophages per area did not significantly differ with WPOI classification, but the mean value tended to be inversely proportional to WPOI classification (Fig. 1E-CD68 and F). In contrast, the numbers of CCL22-positive macrophages per area did not differ in WPOI-1 vs. -2, but increased proportionally from WPOI-2 to -5 (Fig. 1E-CCL22 and G). Fig. 1H shows the CCL22/CD68 ratio (CCL22 ratio). WPOI-high SCC had a significantly high CCL22 ratio (vs. WPOI-low; $\mathrm{P}<0.001$ ). These results suggest that the CCL22 ratio may be an indicator of WPOI classification.

Implications of expression of CCL22 and lymph node relapse for prognosis. Fig. 2A shows H\&E and CCL22 stains for the same grade of WPOI-high cases, with or without lymph node relapse, during the observation period. CCL22-positive macrophage expression was significantly higher in lymph node relapse cases (Fig. 2B). In comparison, CD68 expression showed a slight low mean and median value in patients with lymph node relapse, but this was not significantly different compared to those without lymph node relapse (Fig. 2C). In cases of lymph node relapse, the expression of CD8 positive cells was significantly lower, whereas the expression of Ki67 was significantly higher (Fig. 2D and E).

For WPOI-high patients, the relationship between lymph node relapse and prognosis was examined. As a result, a difference in the 5-year OS rates between patients, with and without lymph node relapse, was statistically significant ( $\mathrm{P}=0.0014$; Fig. 2F).

Relationship between LVD and CCL22 expression via WPOI and DOI. Lymphatic vessels with D2-40 immunohistological staining 

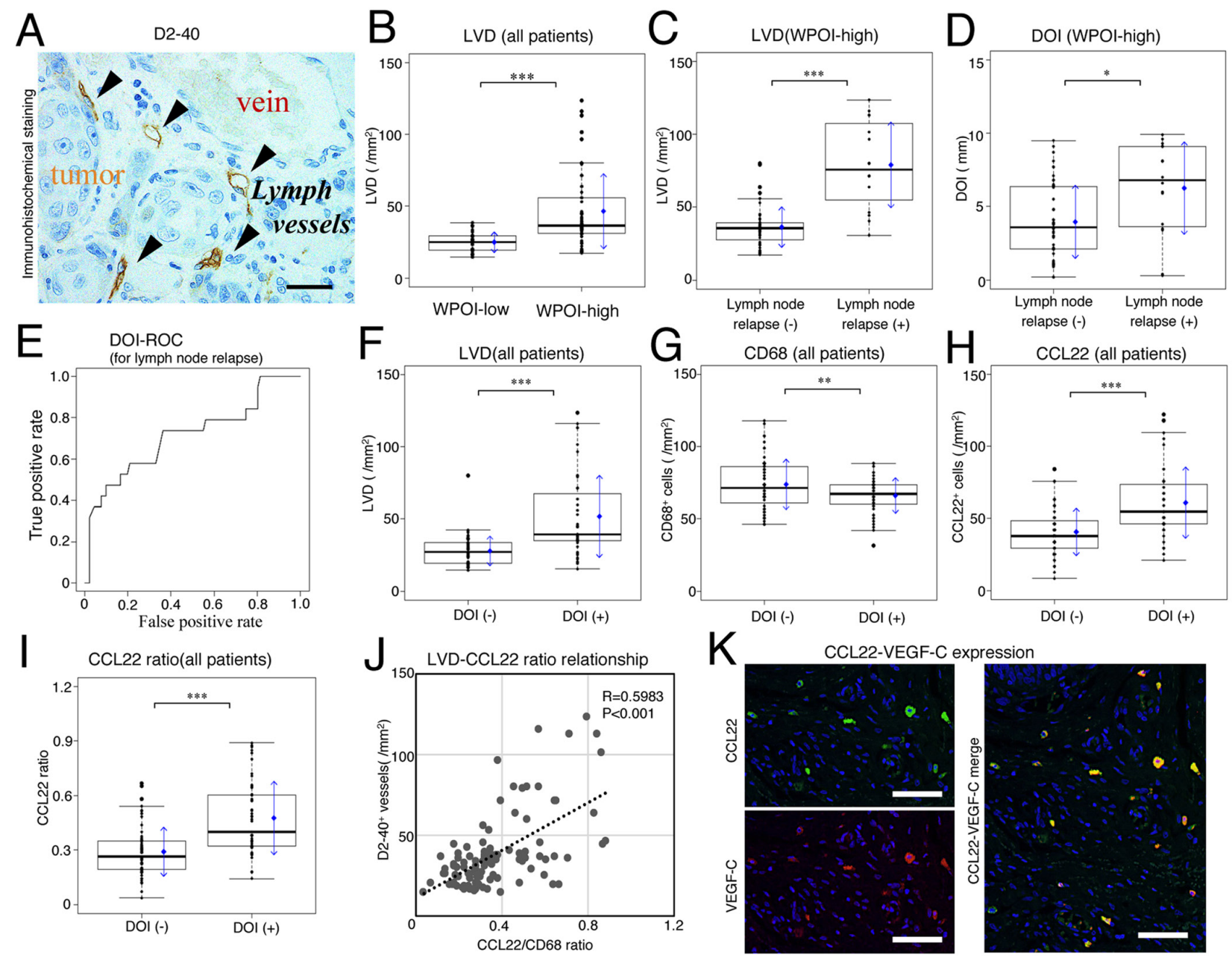

Figure 3. Comparison of LVD and DOI by WPOI grade, lymph node relapse and CCL22 expression. (A) Vessels with positive D2-40 immunohistochemical staining, as well as a vein and tumor tissue (scale bar, $10 \mu \mathrm{m}$ ). Arrowheads indicate lymph vessels. (B) LVD expression was compared between WPOI-1 and -2 (low) and WPOI-3, -4 and -5 (high) lesions. Comparisons of (C) LVD and (D) DOI in patients with lymph node relapse in the WPOI-high group. (E) ROC curve for DOI and lymph node relapse $(3.4 \mathrm{~mm}$ was defined as the cut-off value). Comparisons between the DOI-negative $(<3.4 \mathrm{~mm})$ and -positive $\left(\geq 3.4 \mathrm{~mm}\right.$ ) groups of $(\mathrm{F}) \mathrm{LVD},(\mathrm{G}) \mathrm{CD}^{+} 8^{+}$and $(\mathrm{H})$ CCL22 $2^{+}$macrophages. (I) Association between the CCL22+/CD68 ${ }^{+}$macrophage ratio (CCL22 ratio) and DOI. (J) Correlation between the CCL22 ratio and LVD. (K) Immunofluorescence micrographs of CCL22 (green) and VEGF-C (red) expression on macrophages in the tumor microenvironment (scale bar, $100 \mu \mathrm{m}$ ). In box plots, the boxes display the median and interquartile range of the data, and the whiskers display the 10th and 90 th percentiles. Blue diamonds and blue arrows indicate the mean $\pm \mathrm{SD}$. ${ }^{*} \mathrm{P}<0.05 ;{ }^{* *} \mathrm{P}<0.01 ;{ }^{* * *} \mathrm{P}<0.001$. N.S., not significant; DOI, depth of invasion; LVD, density of lymphatic vessels; ROC, receiver operating characteristic; VEGF, vascular endothelial growth factor; WPOI, worst pattern of invasion; CCL22, C-C motif chemokine ligand 22.

are shown in Fig. 3A. A significant relationship between increased LVD and WPOI was observed (WPOI-low vs. -high; P<0.001; Fig 3B). When LVD was compared in WPOI-high cases, with or without lymph node relapse, it was found to be significantly increased in the lymph node relapse group $(\mathrm{P}<0.001 ;$ Fig. 3C).

Fig. 3D shows the relationship between DOI and lymph node relapse in WPOI-high SCC. DOI was significantly higher in patients with lymph node relapse (without vs. with lymph node relapse; mean, 3.96 vs. $6.26 \mathrm{~mm}, \mathrm{P}=0.0145)$. Therefore, not only WPOI but also DOI seems to be related to LVD.

Fig. 3E shows the ROC curve of DOI for lymph node relapse [area under the curve (AUC); 0.7524]. DOI could be thus classified into two $(<3.4 \mathrm{~mm}$; $\mathrm{n}=63$; and $\geq 3.4 \mathrm{~mm}$; $\mathrm{n}=47$ ) expression groups using the Youden index from the ROC curve (sensitivity, 0.8333; specificity, 0.6522). When comparing between WPOI-low and -high for DOI cut-off values ( $n=5$ and $n=42)$, the positive rate of DOI was significantly higher in the WPOI-high group (Fisher's exact test;
$\mathrm{P}<0.001$ ). Additionally, this cut-off value was significantly correlated with LVDs in all patients (Fig. 3F). The expression of CD68 and CCL22 in TAMs showed opposing trends when comparing negative and positive DOI (Fig. $3 \mathrm{G}$ and H). As a result, the CCL22-positive/CD68-positive macrophage ratio (CCL22 ratio) was most useful as an index for macrophages with a DOI cut-off value for lymph node relapse (Fig. 3I).

A correlation between LVD and the number of CCL22-positive macrophages or the CCL22 ratio was examined. The increase in the LVD was proportional to the expression of both the number of CCL22-positive macrophages $/ \mathrm{mm}^{2}(\mathrm{R}=0.4400 ; \mathrm{P}<0.001)$ and CCL22 ratio $(\mathrm{R}=0.5983$; $\mathrm{P}<0.001$; Fig. 3J), showing a significantly positive correlation.

These results suggest that both WPOI and DOI may be involved in CCL22-mediated lymph node metastasis. Additionally, a reason for the correlation between CCL22 and LVD was thought to be that CCL22-positive macrophages expressed VEGF-C (Fig. 3K). 
Table I. Clinicopathological data of 110 patients with tongue SCC divided in WPOI-1 $(n=19)$, WPOI-2 (n=24), WPOI-3 (n=24), WPOI-4 (n=29) and WPOI-5 (n=14).

\begin{tabular}{|c|c|c|c|c|c|c|}
\hline Parameters & WPOI-1 & WPOI-2 & WPOI-3 & WPOI-4 & WPOI-5 & Total \\
\hline \multicolumn{7}{|l|}{ Age, n (\%) } \\
\hline$\geq 75$ years & $6(31.6)$ & $6(25.0)$ & $7(29.2)$ & $11(37.9)$ & $2(14.3)$ & $32(29.1)$ \\
\hline$<75$ years & $13(68.4)$ & $18(75.0)$ & $17(70.8)$ & $18(62.1)$ & $12(85.7)$ & 78 (70.9) \\
\hline \multicolumn{7}{|l|}{ Sex, n $(\%)$} \\
\hline Male & $13(68.4)$ & $19(79.2)$ & $17(70.8)$ & $20(69.0)$ & $9(64.3)$ & 78 (69.9) \\
\hline Female & $6(31.6)$ & $5(20.8)$ & $7(29.2)$ & $9(31.0)$ & $5(35.7)$ & $32(29.1)$ \\
\hline \multicolumn{7}{|c|}{ Alcohol use, n (\%) } \\
\hline Yes & $12(63.2)$ & $14(58.3)$ & $13(54.2)$ & $18(62.1)$ & $9(64.3)$ & $66(60.0)$ \\
\hline No & $7(36.8)$ & $10(41.7)$ & $11(45.8)$ & $11(37.9)$ & $5(35.7)$ & $44(40.0)$ \\
\hline \multicolumn{7}{|l|}{ Smoking, n (\%) } \\
\hline Yes & $15(78.9)$ & $19(79.2)$ & $20(83.3)$ & $22(75.9)$ & $9(64.3)$ & $85(77.3)$ \\
\hline No & $4(21.1)$ & $5(20.8)$ & $4(16.7)$ & $7(24.1)$ & $5(35.7)$ & $25(22.7)$ \\
\hline \multicolumn{7}{|l|}{ Grade, n (\%) } \\
\hline I & $15(78.9)$ & $18(75.0)$ & $14(58.3)$ & $16(55.2)$ & $3(21.4)$ & $66(60.0)$ \\
\hline II & $4(21.1)$ & $6(25.0)$ & $9(37.5)$ & $10(34.5)$ & $4(28.6)$ & $33(30.0)$ \\
\hline III & $0(0.0)$ & $0(0.0)$ & $1(4.2)$ & $3(10.3)$ & $7(50.0)$ & $11(10.0)$ \\
\hline \multicolumn{7}{|l|}{ DOI, n (\%) } \\
\hline$\geq 5 \mathrm{~mm}$ & $0(0.0)$ & $0(0.0)$ & $4(16.7)$ & $13(44.8)$ & $9(64.3)$ & $26(23.6)$ \\
\hline$<5 \mathrm{~mm}$ & $19(100.0)$ & $24(100.0)$ & $20(83.3)$ & $16(55.2)$ & $5(35.7)$ & $84(76.4)$ \\
\hline Mean DOI, mm & 0.816 & 1.621 & 3.142 & 4.590 & 6.679 & 3.240 \\
\hline \multicolumn{7}{|c|}{ Lymphatic vessel invasion, n (\%) } \\
\hline Yes & $0(0.0)$ & $3(12.5)$ & $12(50.0)$ & $18(62.1)$ & $14(100.0)$ & $47(42.7)$ \\
\hline No & $19(100.0)$ & $21(87.5)$ & $12(50.0)$ & $11(37.9)$ & $0(0.0)$ & $63(57.3)$ \\
\hline \multicolumn{7}{|c|}{ Lymph node relapse, $\mathrm{n}(\%)$} \\
\hline Yes & $1(5.3)$ & $1(4.2)$ & $3(12.5)$ & $5(17.2)$ & $8(57.1)$ & $18(16.4)$ \\
\hline No & $18(94.7)$ & $23(95.8)$ & $21(87.5)$ & $24(82.8)$ & $6(42.9)$ & $92(83.6)$ \\
\hline \multicolumn{7}{|c|}{ Death due to tongue SCC, n (\%) } \\
\hline Yes & $1(5.3)$ & $2(8.3)$ & $2(8.3)$ & $4(13.8)$ & $10(71.4)$ & $19(17.3)$ \\
\hline No & $18(94.7)$ & $22(91.7)$ & $22(91.7)$ & $25(86.2)$ & $4(28.6)$ & $91(82.7)$ \\
\hline
\end{tabular}

DOI, depth of invasion; SCC, squamous cell carcinoma; WPOI, worst pattern of invasion.

Implications of CCL22 ratio for lymph node relapse in patients with WPOI-high SCC. WPOI-high patients had a higher lymph node relapse rate. We therefore examined the predictors for lymph node relapse in this patient group.

We were able to divide the patients into two groups according to the CCL22 ratio as follows: low $(<0.38 ; n=72)$ and high $(\geq 0.38 ; n=38)$ based on ROC curve analysis for lymph node relapse. The AUCs for the number of CCL22/ $/ \mathrm{mm}^{2}$ and CCL22 ratios for lymph node relapse according to the ROC curve were 0.7811 vs. 0.8210 . The AUC was higher for the CCL22 ratio. In patients with high WPOI, predictor variables including grade, DOI, CCL22 ratio, and majority parameters (age, alcohol use, sex, and smoking) were analyzed in a logistic regression model with lymph node recurrence as the dependent variable. Table II shows adjusted OR and $\mathrm{p}$ values. The lymph node relapse in patients with WPOI-high was significantly associated with a high CCL22 ratio $(\mathrm{P}=0.0225)$ but not with the other variables by univariate and multivariate logistic regression model analyses.
Relationship of CCL22 and VEGF-C expression via CCR4 expression. In WPOI-high lesions, the expression of CCR4-positive cells was found to be significantly higher than in WPOI-low lesions (Fig. 4A). In addition, more CCR4-positive cells were observed immunohistologically in patients with lymph node relapse in the same lesion as in Fig. 2A (Fig. 4B). Among WPOI-high cases, CCR4-positive cells were significantly more prevalent in lymph node relapse cases (Fig. 4B) and were found around VEGF-C positive macrophages (Fig. 4C). The aggregation of CCR4-positive cells was proportional to the expression of VEGF-C-positive cells, with a significant correlation noted $(\mathrm{R}=0.6532 ; \mathrm{P}<0.001$; Fig. 4D).

Thus, the function of CCR4-positive cells was also important as a factor in the correlation between CCL22 and VEGF-C.

Regulation of CCL22 and VEGF-C expression via IL-4/STAT6. In vitro, CCL22 expression was significantly 
Table II. Univariate and multivariate logistic regression model used to identify independent predictors for lymph node relapse in WPOI-3, -4 and -5 groups.

\begin{tabular}{|c|c|c|c|c|c|c|c|}
\hline \multirow[b]{2}{*}{ Parameters } & \multirow[b]{2}{*}{$\mathrm{N}(\%)$} & \multicolumn{3}{|c|}{ Univariate analysis } & \multicolumn{3}{|c|}{ Multivariate analysis } \\
\hline & & OR & $95 \% \mathrm{CI}$ & P-value & OR & $95 \% \mathrm{CI}$ & P-value \\
\hline \multicolumn{8}{|l|}{ Age, years } \\
\hline$\geq 75$ & $20(29.9)$ & 0.729 & $0.182-2.474$ & 0.628 & - & - & - \\
\hline$<75$ & $47(70.1)$ & & & & & & \\
\hline \multicolumn{8}{|l|}{ Sex } \\
\hline Female & $21(31.3)$ & 1.500 & $0.445-5.995$ & 0.532 & - & - & - \\
\hline Male & $46(68.7)$ & & & & & & \\
\hline \multicolumn{8}{|l|}{ Alcohol use } \\
\hline Yes & $40(59.7)$ & 1.167 & $0.373-3.889$ & 0.794 & - & - & - \\
\hline No & $27(40.3)$ & & & & & & \\
\hline \multicolumn{8}{|l|}{ Smoking } \\
\hline Yes & $51(76.1)$ & 0.407 & $0.119-1.430$ & 0.150 & - & - & - \\
\hline No & $16(23.9)$ & & & & & & \\
\hline \multicolumn{8}{|l|}{ Grade } \\
\hline III & $11(16.4)$ & 9.139 & $2.298-41.605$ & 0.002 & 3.408 & $0.751-17.575$ & 0.120 \\
\hline I or II & $56(83.6)$ & & & & & & \\
\hline \multicolumn{8}{|l|}{ DOI, mm } \\
\hline$\geq 3.4$ & $42(62.7)$ & 5.750 & $1.414-39.059$ & 0.030 & 4.251 & $0.871-32.042$ & 0.100 \\
\hline$<3.4$ & $25(37.3)$ & & & & & & \\
\hline \multicolumn{8}{|c|}{ CCL22/CD68 ratio } \\
\hline$\geq 0.38$ & $35(52.2)$ & 21.429 & $3.885-402.398$ & 0.004 & 12.736 & $2.018-250.014$ & 0.023 \\
\hline$<0.38$ & $32(47.8)$ & & & & & & \\
\hline
\end{tabular}

DOI, depth of invasion; OR, odds ratio; WPOI, worst pattern of invasion; CCL22, C-C motif chemokine ligand 22.

higher in M2-like compared to M1-like macrophages, as was VEGF-C expression; in M2-like macrophages, the expression of both was increased by IL-4 in a dose-dependent manner (Fig. 4E and F). The regulation of VEGF-C expression in macrophages is known to be via an IL-4/STAT6 signaling pathway (31); CCL22 was also examined in this study. We found that W1 and W2 promotors, including a STAT6 motif, had significantly higher luciferase activity after IL-4 stimulation. However, there was no significant difference in the luciferase activities of W3 and W4 promoters without a STAT6 motif after IL-4 stimulation. When a STAT6 mutation was inserted into W1 (M1), the luciferase activity significantly decreased (Fig. 4G) and no longer responded to IL-4 stimulation. STAT6 activation also affected the production of CCL22 protein via IL-4 (Fig. 4H). Our results supported the conclusion that IL-4 induces CCL22 expression via an STAT6-dependent pathway. Fig. 4I shows STAT6 activated-cells (green) and VEGF-C-positive macrophages (red). VEGF-C-positive cells accompanied STAT6 activation. In addition, STAT6 activation of almost all carcinoma cells was observed in the deeply invaded part of the tumor. However, in normal tissue around the tumor, STAT6 activation was observed only in basal cells (Fig. 4I normal).

Thus, STAT6 activation was considered to be an important factor in lymph node metastasis of tongue SCC.

\section{Discussion}

Immunosuppressive and anti-inflammatory molecules linked to TAMs are associated with a poor cancer prognosis $(32,33)$. Interactions between cells in the tumor microenvironment are critical for tumor progression and are involved in invasion and metastasis. Certainly, macrophages are also involved in the interaction (34). In this study, we focused on lymphangiogenesis through cell-cell interactions in which M2-like TAMs attract Th2 cells in the tumor microenvironment.

In patients with tongue SCC, having a WPOI-high grade classification generally led to a poor prognosis (23). The WPOI classification was divided into two groups according to the lymph node relapse ratio. A WPOI-high group, involving invasive tumor islands, showed a significant decrease in CD8 and increase in $\mathrm{Ki} 67$, and a significantly poor prognosis. The expression of CCL22 in the tumor microenvironment also correlated with decreased CD8 and increased Ki67 in our previous study (6). The molecular biological effects of CCL22 include several factors that influence tumor prognosis. However, few studies have investigated the correlation between CCL22 expression and lymph node metastasis. Lymph node relapse and lymphatic vessel invasion are important prognostic determinants of OSCC. However, venous invasion did not correlate with prognosis in patients with early tongue 
A

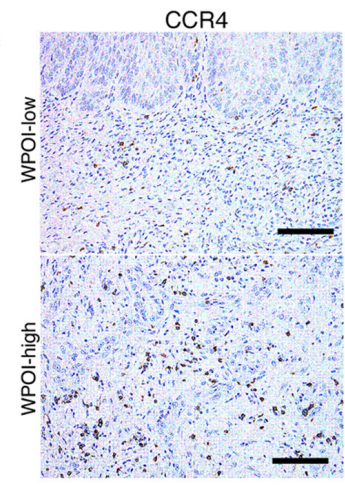

C

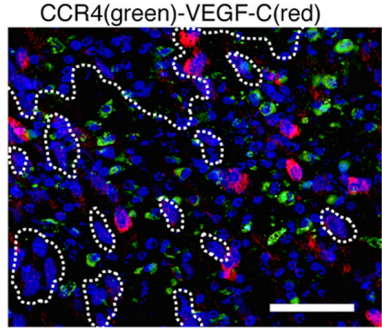

G THP-1 cell relative luciferase activation

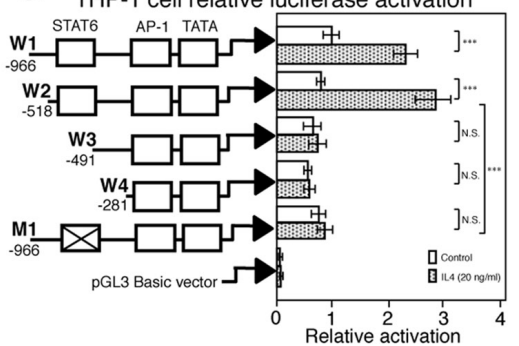

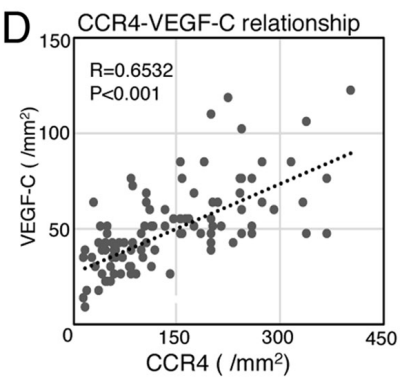

CCR4

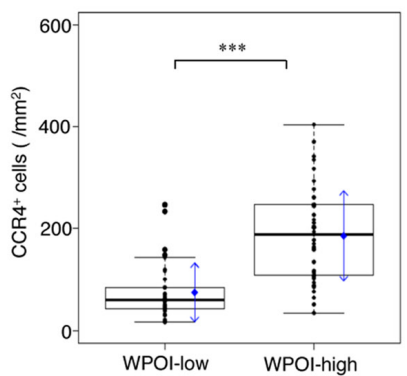

$\mathrm{H}$

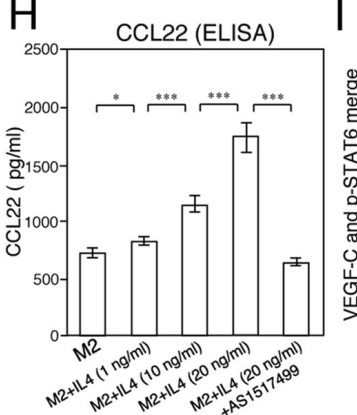

$\mathrm{B}$
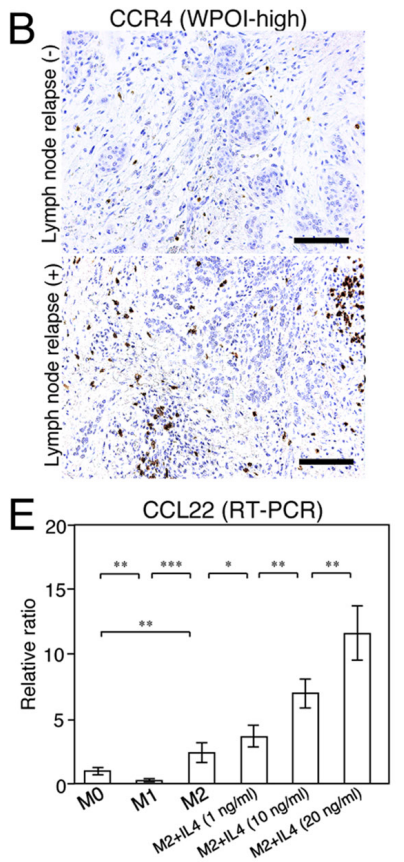

CCR4 (WPOI-high)
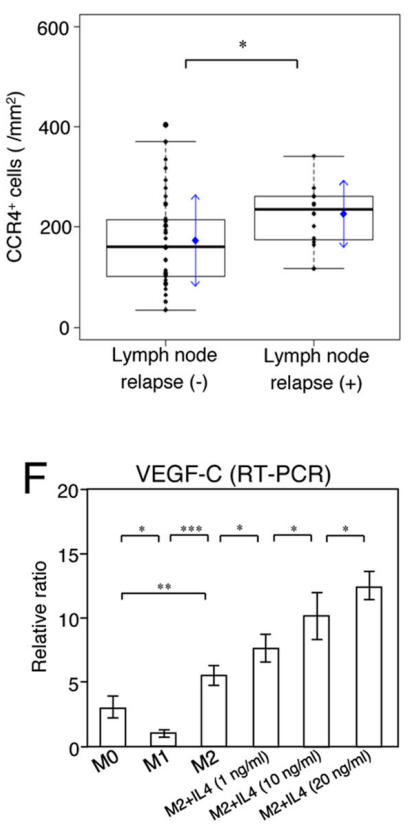

VEGF-C(red) and phospho-STAT6 (green)

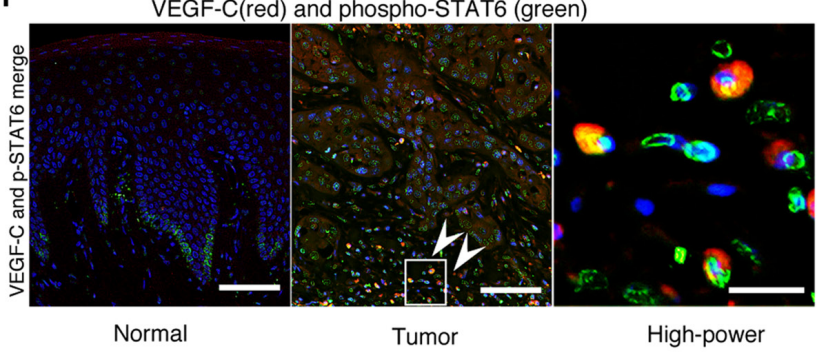

Figure 4. Association between CCL22 and CCR4 via the IL-4/STAT6 signaling pathway. Comparisons of CCR4 expression in (A) WPOI-low and -high lesions, and (B) WPOI-high tissues with or without lymph node relapse (scale bar, $100 \mu \mathrm{m}$ ). In box plots, the boxes display the median and interquartile range of the data, and the whiskers display the 10th and 90th percentiles. Blue diamonds and blue arrows indicate the mean \pm SD. (C) Localization of VEGF-C and CCR $4^{+}$cells. VEGF-C ${ }^{+}$cells appeared surrounding the invasive tumor and CCR4 $4^{+}$cells were observed within their vicinity. The dotted line outlines the boundary of the tumor and invading tumor cells. The blue DAPI stain indicates cellular nuclei (scale bar, $50 \mu \mathrm{m}$ ). (D) Correlation between CCR4 and VEGF-C expression. Macrophage subtypes and the effect of IL-4 stimulation on (E) CCL22 and (F) VEGF-C expression were examined using human macrophages. (G) Luciferase assay in THP-1 cells with 5'-serial deletion constructs and point mutations, and responses to $20 \mathrm{ng} / \mathrm{ml}$ IL-4. The luciferase activity of each sample was normalized to $\beta$-galactosidase activity. The ' $\mathrm{X}$ ' in the box indicates a mutation. (H) CCL22 levels in M2-like macrophages in response to IL-4 $(1,10$ and $20 \mathrm{ng} / \mathrm{ml})$ and AS1517499 $(200 \mathrm{nM})$ in cell culture supernatants measured by ELISA. (I) Activation of IL-4/STAT6 and VEGF-C expression in a case of WPOI-4 with lymph node relapse. The image labeled 'Normal' shows normal tissue away from the tumor, while the image labeled 'Tumor' shows a deep infiltration site (scale bar, $200 \mu \mathrm{m}$ ). The 'High-power' image shows a high-power magnification image of the square indicated by arrowheads in the 'Tumor' image (scale bar, $20 \mu \mathrm{m}$ ). Phospho-STAT6 (green) was expressed in the nucleus and VEGF-C (red) was expressed in the cytoplasm. The blue DAPI stain indicates cellular nuclei. The data are expressed as the mean \pm SD of triplicates from three independent experiments. ${ }^{*} \mathrm{P}<0.05 ;{ }^{* * *} \mathrm{P}<0.01 ;{ }^{* * * *} \mathrm{P}<0.001$. N.S., not significant; IL-4, interleukin-4; p/phospho-STAT6, phosphorylated signal transducer and activator of transcription 6; VEGF, vascular endothelial growth factor; WPOI, worst pattern of invasion; CCL22, C-C motif chemokine ligand 22.

SCC (6). Lymphangiogenesis is one of the causes of lymph node metastasis via lymph node invasion and leads to a poor prognosis (35).

Compared with WPOI-low, the WPOI-high group showed increased expression of CCL22 and LVD. WPOI-high with lymph node relapse also showed a further increase in expression of CCL22, LVD, and DOI. When the correlation between the CCL22 ratio and LVD was examined, a positive and significant correlation was found. In this study, the cut-off value of DOI for lymph node recurrence was defined as $3.4 \mathrm{~mm}$, which was also consistent with the cut-off DOI value for positive sentinel lymph node metastasis in OSCC (25). In future, the DOI cut-off value is expected to be a reference value for lymph node metastasis in early OSCC.
IL-4 is a typical Th2 cytokine that differentiates T lymphocytes predominantly into Th2 cells, and also differentiates macrophages into M2-like macrophages (19). M2-like macrophages produced CCL22 and VEGF-C, and the response was enhanced by IL-4. An IL-4 dominant environment also allows the migration of macrophages into the local environment and, consequently, TAMs differentiate into M2-like macrophages $(36,37)$. Moreover, lymphatic vessels proliferate when the cytokine balance is predominantly Th2 (38). IL-4, in cooperation with tumor cells and macrophages, has various roles in the tumor microenvironment (36). Interestingly, the proliferation of lymphatic endothelial cells is known to be suppressed by Th2 cytokines (39), supporting the notion that the effect of IL-4 on lymphangiogenesis in the tumor microenvironment 
depends on cell-cell interactions via the M2-like differentiation of TAMs. Macrophage-mediated cell-cell interaction is thought to be important, as seen in lymphangiogenesis due to increased VEGF-C in a Th2-dominant environment in other diseases $(38,40)$. The tumor microenvironment contains many immune or inflammatory cells, such as M1-like macrophages and Th1 cells. The proportion of M2-like macrophages, but not the total number of CD68-positive macrophages, is associated with the presence of lymph node relapse. Therefore, the balance of each type of inflammatory cell may affect the extent of tumor progression via cell-cell interactions (41).

Macrophage CCL22 expression is also dependent on the IL-4/STAT6 signaling pathway, which is generally known as a pathway for the differentiation of $\mathrm{T}$ cells into $\mathrm{Th} 2$ as shown in animal experimental models (42). In this study, the activation of the IL-4/STAT6 signaling pathway in TAMs led to the expression of CCL22 and VEGF-C in the tumor microenvironment of tongue SCC via a Th2-predominant environment. The activation of this pathway may play a critical role in the tumor progression response via CCL22 expression in the tumor microenvironment. However, a quantitative correlation was not found between the number of positive lymph vessels for VEGF receptor-3 (VEGFR3), a VEGF-C receptor, and lymph node relapse (data not shown). Since the expression of VEGFR3 has been demonstrated in many tumor and immune cells, activation of signal transduction pathways, in addition to VEGFR3-expressing cell types, may be important for lymph node relapse (43). Further examination of various quantitative and qualitative parameters of VEGFR3 is considered necessary in future.

In conclusion, WPOI and DOI were revealed to be useful parameters for lymph node relapse in patients with tongue SCC. It is suggested that CCL22 contributes to the role of M2-like differentiated TAMs in prognosis and lymph node relapse via IL-4/STAT6 and VEGF. The IL-4/STAT6 signaling pathway may be a new molecular target for tongue SCC, as shown in other cancers (44).

\section{Acknowledgements}

The authors would like to thank Professor Sato Hiroaki (Department of Forensic Medicine, School of Medicine, University of Occupational and Environmental Health, Kitakyushu, Japan) for the useful discussion with respect to the writing of the manuscript.

\section{Funding}

The present study was financially supported by a grant (grant no. H28-031202) from the University of Occupational and Environmental Health (Kitakyushu, Japan).

\section{Availability of data and materials}

The datasets used and/or analyzed during the current study are available from the corresponding author on reasonable request.

\section{Authors' contributions}

SK and TN designed the experiments and confirm the authenticity of all the raw data. SK and HN collected the patient samples. SK, HN and UN performed the experiments. SK,
HN, UN and TN analyzed the data and wrote the manuscript. All authors revised, edited, read and approved the final manuscript.

\section{Ethics approval and consent to participate}

Ethics approval for the present study was obtained from the Ethics Committee of the University of Occupational and Environmental Health (Kitakyushu, Japan). Considering the retrospective nature of the protocol for patients with tongue squamous cell carcinoma, which involved using only already existing medical data and specimens that were previously anonymized with no impact on patient care, no specific written informed consent was required by the Ethics Committee. However, blood donors provided written informed consent. The study was performed in accordance with the Declaration of Helsinki.

\section{Patient consent for publication}

Not applicable.

\section{Competing interests}

The authors declare that they have no competing interests.

\section{References}

1. Siegel RL, Miller KD and Jemal A: Cancer statistics, 2015. CA Cancer J Clin 65: 5-29, 2015.

2. Warnakulasuriya S: Global epidemiology of oral and oropharyngeal cancer. Oral Oncol 45: 309-316, 2009.

3. Ariyoshi Y, Shimahara M, Omura K, Yamamoto E, Mizuki H, Chiba H, Imai Y, Fujita S, Shinohara M and Seto K; Japanese Society of Oral and Maxillofacial Surgeons, 2002: Epidemiological study of malignant tumors in the oral and maxillofacial region: Survey of member institutions of the Japanese Society of Oral and Maxillofacial Surgeons, 2002. Int J Clin Oncol 13: 220-228, 2008.

4. Dünne AA, Müller HH, Eisele DW, Kessel K, Moll R and Werner JA: Meta-analysis of the prognostic significance of perinodal spread in head and neck squamous cell carcinomas (HNSCC) patients. Eur J Cancer 42: 1863-1868, 2006.

5. Afzali P and Ward BB: Management of the neck in oral squamous cell carcinoma: Background, classification, and current philosophy. Oral Maxillofac Surg Clin North Am 31: 69-84, 2019.

6. Kimura S, Nanbu U, Noguchi H, Harada Y, Kumamoto K, Sasaguri Y and Nakayama T: Macrophage CCL22 expression in the tumor microenvironment and implications for survival in patients with squamous cell carcinoma of the tongue. J Oral Pathol Med 48: 677-685, 2019.

7. Kaesler S, Wölbing F, Kempf WE, Skabytska Y, Köberle M, Volz T, Sinnberg T, Amaral T, Möckel S, Yazdi A, et al: Targeting tumor-resident mast cells for effective anti-melanoma immune responses. JCI Insight 4: e125057, 2019.

8. Messex JK, Byrd CJ and Liou GY: Signaling of macrophages that contours the tumor microenvironment for promoting cancer development. Cells 9: e919, 2020.

9. GodiskaR,Chantry D,RaportCJ,Sozzani S,Allavena P,Leviten D, Mantovani A and Gray PW: Human macrophage-derived chemokine (MDC), a novel chemoattractant for monocytes, monocyte-derived dendritic cells, and natural killer cells. J Exp Med 185: 1595-1604, 1997.

10. Imai T, Chantry D, Raport CJ, Wood CL, Nishimura M, Godiska R, Yoshie O and Gray PW: Macrophage-derived chemokine is a functional ligand for the CC chemokine receptor 4. J Biol Chem 273: 1764-1768, 1998.

11. Curiel TJ, Coukos G, Zou L, Alvarez X, Cheng P, Mottram P, Evdemon-Hogan M, Conejo-Garcia JR, Zhang L, Burow M, et al: Specific recruitment of regulatory $\mathrm{T}$ cells in ovarian carcinoma fosters immune privilege and predicts reduced survival. Nat Med 10: 942-949, 2004. 
12. Imai T, Nagira M, Takagi S, Kakizaki M, Nishimura M, Wang J, Gray PW, Matsushima K and Yoshie O: Selective recruitment of CCR4-bearing Th2 cells toward antigen-presenting cells by the $\mathrm{CC}$ chemokines thymus and activation-regulated chemokine and macrophage-derived chemokine. Int Immunol 11: 81-88, 1999.

13. Biedermann T, Schwärzler C, Lametschwandtner G, Thoma G Carballido-Perrig N, Kund J, de Vries JE, Rot A and Carballido JM: Targeting CLA/E-selectin interactions prevents CCR4-mediated recruitment of human Th2 memory cells to human skin in vivo. Eur J Immunol 32: 3171-3180, 2002.

14. Franciszkiewicz K, Boissonnas $\mathrm{A}$, Boutet $\mathrm{M}$, Combadière $\mathrm{C}$ and Mami-Chouaib F: Role of chemokines and chemokine receptors in shaping the effector phase of the antitumor immune response. Cancer Res 72: 6325-6332, 2012

15. Zwaans BM and Bielenberg DR: Potential therapeutic strategies for lymphatic metastasis. Microvasc Res 74: 145-158, 2007.

16. Moussai D, Mitsui H, Pettersen JS, Pierson KC, Shah KR, Suárez-Fariñas M, Cardinale IR, Bluth MJ, Krueger JG, Carucci JA, et al: The human cutaneous squamous cell carcinoma microenvironment is characterized by increased lymphatic density and enhanced expression of macrophage-derived VEGF-C. J Invest Dermato 131: 229-236, 2011.

17. Nakagawa T, Ohnishi K, Kosaki Y, Saito Y, Horlad H, Fujiwara Y, Takeya M and Komohara Y: Optimum immunohistochemical procedures for analysis of macrophages in human and mouse formalin fixed paraffin-embedded tissue samples. J Clin Exp Hematop 57: 31-36, 2017.

18. Yamagata Y, Tomioka H, Sakamoto K, Sato K, Harada H, Ikeda T and Kayamori K: CD163-positive macrophages within the tumor stroma are associated with lymphangiogenesis and lymph node metastasis in oral squamous cell carcinoma. J Oral Maxillofac Surg 75: 2144-2153, 2017.

19. Kimura S, Noguchi H, Nanbu U, Wang KY, Sasaguri Y and Nakayama T: Relationship between CCL22 expression by vascular smooth muscle cells and macrophage histamine receptors in atherosclerosis. J Atheroscler Thromb 25: 1240-1254, 2018.

20. Tsujikawa T, Yaguchi T, Ohmura G, Ohta S, Kobayashi A, Kawamura N, Fujita T, Nakano H, Shimada T, Takahashi T, et al: Autocrine and paracrine loops between cancer cells and macrophages promote lymph node metastasis via CCR4/CCL22 in head and neck squamous cell carcinoma. Int J Cancer 132: 2755-2766, 2013.

21. Almangush A, Bello IO, Keski-Säntti H, Mäkinen LK, Kauppila JH, Pukkila M, Hagström J, Laranne J, Tommola S, Nieminen O, et al: Depth of invasion, tumor budding, and worst pattern of invasion: Prognostic indicators in early-stage oral tongue cancer. Head Neck 36: 811-818, 2014.

22. Brandwein-Gensler M, Teixeira MS, Lewis CM, Lee B, Rolnitzky L, Hille JJ, Genden E, Urken ML and Wang BY: Oral squamous cell carcinoma: Histologic risk assessment, but not margin status, is strongly predictive of local disease-free and overall survival. Am J Surg Pathol 29: 167-178, 2005.

23. Shimizu S, Miyazaki A, Sonoda T, Koike K, Ogi K, Kobayashi JI, Kaneko T, Igarashi T, Ueda M, Dehari H, et al: Tumor budding is an independent prognostic marker in early stage oral squamous cell carcinoma: With special reference to the mode of invasion and worst pattern of invasion. PLoS One 13: e0195451, 2018.

24. Caldeira PC, Soto AML, de Aguiar MCF and Martins CC: Tumor depth of invasion and prognosis of early-stage oral squamous cel carcinoma: A meta-analysis. Oral Dis 26: 1357-1365, 2020.

25. den Toom IJ, Janssen LM, van Es RJJ, Karagozoglu KH, de Keizer B, van Weert S, Willems SM, Bloemena E, Leemans CR and de Bree R: Depth of invasion in patients with early stage oral cancer staged by sentinel node biopsy. Head Neck 41: 2100-2106, 2019.

26. Amin MB, Edge SB, Greene FL, Byrd DR, Brookland RK, Washington MK, Gershenwald JE, Compton CC, Hess KR, Sullivan DC, et al (eds): AJCC Cancer Staging Manual. 8th edition. Springer, New York, NY, 2017.
27. Lydiatt WM, Patel SG, O'Sullivan B, Brandwein MS, Ridge JA, Migliacci JC, Loomis AM and Shah JP: Head and Neck cancers-major changes in the American Joint Committee on cancer eighth edition cancer staging manual. CA Cancer J Clin 67: 122-137, 2017.

28. Audet N, Beasley NJ, MacMillan C, Jackson DG, Gullane PJ and Kamel-Reid S: Lymphatic vessel density, nodal metastases, and prognosis in patients with head and neck cancer. Arch Otolaryngol Head Neck Surg 131: 1065-1070, 2005.

29. Kimura S, Tanimoto A, Wang KY, Shimajiri S, Guo X, Tasaki T, Yamada S and Sasaguri Y: Expression of macrophage-derived chemokine (CCL22) in atherosclerosis and regulation by histamine via the $\mathrm{H} 2$ receptor. Pathol Int 62: 675-683, 2012.

30. Kanda Y: Investigation of the freely available easy-to-use software 'EZR' for medical statistics. Bone Marrow Transplant 48: 452-458, 2013

31. D'Alessio S, Correale C, Tacconi C, Gandelli A, Pietrogrande G, Vetrano S, Genua M, Arena V, Spinelli A, Peyrin-Biroulet L, et al: VEGF-C-dependent stimulation of lymphatic function ameliorates experimental inflammatory bowel disease. J Clin Invest 124: 3863-3878, 2014

32. Komohara Y, Fujiwara Y, Ohnishi K and Takeya M: Tumor-associated macrophages: Potential therapeutic targets for anti-cancer therapy. Adv Drug Deliv Rev 99: 180-185, 2016

33. Miyasato Y, Takashima Y, Takeya H, Yano H, Hayano A, Nakagawa T, Makino K, Takeya M, Yamanaka R and Komohara Y: The expression of PD-1 ligands and IDO1 by macrophage/ microglia in primary central nervous system lymphoma. J Clin Exp Hematop 58: 95-101, 2018.

34. Komohara Y and Takeya M: CAFs and TAMs: Maestros of the tumour microenvironment. J Pathol 241: 313-315, 2017.

35. Alitalo K, Tammela T and Petrova TV: Lymphangiogenesis in development and human disease. Nature 438: 946-953, 2005.

36. Shi VY, Bao L and Chan LS: Inflammation-driven dermal lymphangiogenesis in atopic dermatitis is associated with $\mathrm{CD}_{11 \mathrm{~b}^{+}}$ macrophage recruitment and VEGF-C up-regulation in the IL-4-transgenic mouse model. Microcirculation 19: 567-579, 2012.

37. Roca H, Varsos ZS, Sud S, Craig MJ, Ying C and Pienta KJ: CCL2 and interleukin- 6 promote survival of human $\mathrm{CD} 11 \mathrm{~b}^{+}$peripheral blood mononuclear cells and induce M2-type macrophage polarization. J Biol Chem 284: 34342-34354, 2009.

38. Zhang B, Yao G, Zhang Y, Gao J, Yang B, Rao Z, Gao J and Gao J: M2-polarized tumor-associated macrophages are associated with poor prognoses resulting from accelerated lymphangiogenesis in lung adenocarcinoma. Clinics (São Paulo) 66: 1879-1886, 2011.

39. Savetsky IL, Ghanta S, Gardenier JC, Torrisi JS, García Nores GD, Hespe GE, Nitti MD, Kataru RP and Mehrara BJ: Th2 cytokines inhibit lymphangiogenesis. PLoS One 10: e0126908, 2015.

40. Zhang B, Wang J, Gao J, Guo Y, Chen X, Wang B, Gao J, Rao Z and Chen Z: Alternatively activated RAW264.7 macrophages enhance tumor lymphangiogenesis in mouse lung adenocarcinoma. J Cell Biochem 107: 134-143, 2009.

41. Hinshaw DC and Shevde LA: The tumor microenvironment innately modulates cancer progression. Cancer Res 79: 4557-4566, 2019.

42. Fulkerson PC, Zimmermann N, Hassman LM, Finkelman FD and Rothenberg ME: Pulmonary chemokine expression is coordinately regulated by STAT1, STAT6, and IFN-gamma. J Immunol 173: 7565-7574, 2004

43. Hsu MC, Pan MR and Hung WC: Two birds, one stone: Double hits on tumor growth and lymphangiogenesis by targeting vascular endothelial growth factor receptor 3. Cells 8: e270, 2019.

44. Binnemars-Postma K, Bansal R, Storm G and Prakash J: Targeting the Stat6 pathway in tumor-associated macrophages reduces tumor growth and metastatic niche formation in breast cancer. FASEB J 32: 969-978, 2018.

This work is licensed under a Creative Commons Attribution-NonCommercial-NoDerivatives 4.0 International (CC BY-NC-ND 4.0) License. 